Canadian Oncology

Nursing Journal

Revue canadienne

de soins infirmiers

en oncologie

Volume 27, Issue 1 • Winter 2017

elSSN: $2368-8076$ 


\title{
S'engager auprès des patients qui veulent mourir : interprétation, présence et contrainte
}

\author{
par David Kenneth Wright, Marina Chirchikova, Véronique Daniel, Vasiliki Bitzas, James Elmore et Marie-Laurence Fortin
}

\section{RÉSUMÉ}

Les soins de fin de vie changent au Canada. À la lumière des récentes modifications législatives sur l'aide à mourir (euthanasie et suicide assisté), il $y$ a lieu d'examiner le point de vue des infirmières sur les soins à donner aux patients qui veulent mourir. Cette étude descriptive et qualitative rapporte les résultats d'entrevues réalisées auprès de 11 infirmières en oncologie sur leur engagement auprès de patients qui veulent mourir. Les résultats offrent d'importants éléments sur la façon dont les infirmières en oncologie interprètent le désir de mort des patients et sur la présence thérapeutique qu'elles exercent auprès d'eux. De plus, ces résultats témoignent des forces contextuelles qui entravent l'engagement thérapeutique. Les entrevues ont été réalisées avant que les lois changent au Canada, mais offrent des perspectives pertinentes et plus que jamais applicables, car le nombre de patients qui songent et demandent à être assistés dans la mort ira en s'accroissant dans le nouveau paysage des soins prodigués à ce chapitre au pays.

$\mathrm{L}$ 'euthanasie implique que l'on mette fin délibérément à la vie d'un patient pour des raisons de compassion, à sa demande expresse. Le suicide assisté veut que l'on fournisse

\section{AU SUJET DES AUTEURS}

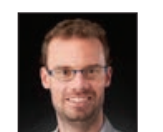

David Kenneth Wright, Ph.D., inf. aut., ICSP(C), École des sciences infirmières, Université d'Ottawa, Ottawa, Centre de recherche de St. Mary, Montréal

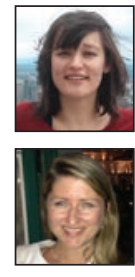

Marina Chirchikova, M.Sc.(A), inf. aut., Écoles des sciences infirmières Ingram, Université McGill, Montréal

Véronique Daniel, M.Sc.(A), inf. aut., Écoles des sciences infirmières Ingram, Université McGill, Montréal

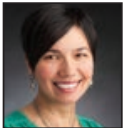

Vasiliki Bitzas, Ph.D., inf. aut., ICSP(C), Hôpital général juif, Montréal

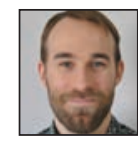

James Elmore, M.Sc.(A), inf. aut., Centre de recherche de St. Mary, Montréal, Écoles des sciences infirmières Ingram, Université McGill, Montréal

Marie-Laurence Fortin, M.Sc.inf., inf. aut., ICSP(C), Hôpital général juif, Montréal

Auteur-ressource : David Kenneth Wright, Ph.D., inf. aut., CHPCN(C), Professeur adjoint, École des sciences infirmières, Faculté des sciences de la santé, Université d'Ottawa, Pavillon Roger-Guindon, 3247A, 451 Smyth Road, Ottawa (Ontario), K1H 8 M5 Canada

Tél. : 613-562-5800, poste 8533; Téléc. : 613-562-5443

dk.wright@uottawa.ca

DOI: $10.5737 / 236880762716573$ au patient les moyens qui lui permettront de mourir, soit des medicaments autoadministrés. Prises collectivement, ces deux pratiques constituent l'aide à mourir (Van der Mass, van Delden, Pignenborg et Looman, 1991). En juin 2014, la province de Québec a légalisé l'euthanasie (sous la désignation « aide médicale à mourir »); la loi est entrée en vigueur le 10 décembre 2015 (Publications Québec, 2016). En avril 2016, le Parlement du Canada, en réponse à la décision de la Cour suprême dans le jugement Carter sur la décriminalisation de l'euthanasie et du suicide assisté (Carter c. Canada, 2015), a adopté la loi C-14. Cette loi, connue sous l'appellation « aide médicale à mourir », en référence à l'euthanasie et au suicide assisté, rend l'euthanasie et le suicide assisté légaux dans l'ensemble des provinces et territoires canadiens (Government of Canada, 2016). Par conséquent, la société canadienne est à l'aube de transformations importantes dans la façon dont les soins de fin de vie seront offerts et vécus.

Dans les pays et territoires ayant légalement régi la mort assistée, les lois ont pour habitude de préciser que ce sont les médecins, et non les infirmières, qui prescrivent (dans le cas du suicide assisté) ou dirigent (dans le cas de l'euthanasie) la mort assistée. Au Canada, toutefois, les infirmières praticiennes ont l'autorisation légale de prescrire et d'administrer des médicaments mortels dans un contexte de mort assistée (Parlement du Canada, 2016). Cela fait du Canada le premier et seul pays au monde où des infirmières peuvent autoriser la mort assistée dans le cadre de leur pratique de soins de fin de vie.

Or, même si cette autorité à fournir une mort assistée se limite aux infirmières praticiennes, d'autres infirmières jouent un rôle important auprès de patients envisageant la mort assistée et la recevant dans leurs soins de fin de vie. Offrir des soins de fin de vie est un aspect universel de la pratique infirmière (Jacono et al., 2009), y compris en oncologie (Wright et Pugnaire-Gros, 2012). Les infirmières tissent au quotidien des liens uniques avec les patients et leur famille, et de ce contexte émergent des idées importantes pour alimenter une prise de décision éthique (Bergum, 2013; Doane et Varcoe, 2007; Nortvedt, 2001; Wright et Brajtman, 2011). Les infirmières partagent en outre une proximité émotionnelle avec eux sur les soins de fin de vie (Barnard, Hollingum, et Hartfiel, 2006; Mok et Chiu, 2004; Raus et al., 2014; Roberts et Snowball, 1999) et pourraient bien s'avérer les premières professionnelles de la santé à qui un patient confiera son désir de mourir (De Bal, Dierckx de Casterle, De Beer, et Gastmans, 2006; De Beer, Gastmans, et Dierckx de Casterle, 2004).

Comme l'a soulignée l'Association des infirmières et infirmiers du Canada (AIIC/CNA) dans le dossier présenté au Comité externe sur les options de réponse législative à Carter c. Canada : « Le personnel infirmier, en raison de ses aptitudes essentielles en interprétation, est vital au processus d'évaluation d'un patient 
exigeant l'AMM [aide médicale à mourir] et doit faire partie du processus décisionnel du patient [...] Il peut aider les patients à explorer leurs sentiments et opinions, à discuter des options/ solutions de rechange et à expliquer le processus d'AMM. » (Association des infirmières et infirmiers du Canada, 2015, p. 6). L'AIIC souligne d'ailleurs que les infirmières feront face dans l'avenir à quantité de risques psychologiques, moraux et légaux qu'il faudra légalement reconnaître et alléger.

Voilà pourquoi un besoin pressant de mieux comprendre la perspective infirmière sur le désir de mort chez les patients en phase terminale se fait sentir. Cela dit, dans les pays et territoires ayant légalisé la mort assistée, l'ensemble de la littérature empirique et éthique traitant du vécu infirmier sur cette pratique est minime (Denier, Dierckx de Casterle, De Bal et Gastmans, 2009, 2010; Denier, Gastmans, De Bal et Dierckx de Casterle, 2010; Dierckx de Casterle, Denier, De Bal et Gastmans, 2010; van de Scheur et van der Arend, 1998). Dans les pays n'ayant pas légalisé la mort assistée, mais où elle est sérieusement débattue, la voix des infirmières peine à se faire entendre dans les débats (Woods et Ascher, 2015).

La perspective des infirmières sur les diverses façons de mourir et d'y réagir est régie par des « convictions sur ce qui est moralement bon » (Cronqvist, Theorell, Burns et Lutzen, 2004, p. 68, traduction). Même si recourir à des cadres déontologiques ou utilitaires, empruntés à la philosophie morale, aide les infirmières dans leurs délibérations éthiques sur les soins de fin de vie, utiliser des cadres relationnels centrés sur les liens interpersonnels qu'offrent de tels soins est tout aussi important (Wright, Brajtman et Bitzas, 2009). Léthique relationnelle est l'un de ces cadres tenant compte « du type de relation qui mène à la croissance du bien » (Bergum, 2004, p. 487, traduction). Comme l'indique Bergum (2013), lorsqu'on adopte pour prémisse que l'espace relationnel est le lieu où la moralité se forge, les considérations éthiques deviennent omniprésentes dans la pratique infirmière et surviennent dans « chaque cas, chaque rencontre et avec chaque patient » (p. 127). Selon Austin (2011), la recherche en éthique relationnelle a démontré de manière répétée l'importance d'un engagement authentique et $\mathrm{du}$ lien dans ces rencontres. Le fait de reconnaître l'authenticité des rencontres éthiques permet, selon Austin, de résister à la mentalité de « service à la clientèle », résultante d'une culture de soins de santé corporatiste. Austin regrette ce glissement vers l'inauthenticité dans les relations de soins de santé, une menace à notre discipline, et il nous invite à réaffirmer le vrai dans la pratique infirmière. Pour Austin, « les vraies infirmières s'engagent de manière sincère auprès de leurs malades, car elles voient les patients et leurs familles comme de vrais êtres humains qu'elles côtoient dans la réalité et en qui elles reconnaissent tout un éventail de possibilités » (p. 163, traduction, nous soulignons). Nous prenons cette emphase normative sur l'engagement authentique dans les relations infirmières comme point de départ d'une étude sur les « vrais » liens thérapeutiques que tissent les infirmières avec les patients atteints de cancer et qui envisagent la mort avec l'aide des soins de fin de vie.

Voici la question de recherche qui a guidé l'étude : Comment les infirmières en oncologie décrivent-elles leur engagement auprès des patients préoccupés par le désir de mourir? Notre objectif était d'explorer la manière dont les infirmières en oncologie comprennent les patients atteints de cancer en phase terminale et envisageant la mort, et la manière dont elles réagissent au désir de mort de ces patients, pour mieux saisir leur point de vue sur les liens thérapeutiques en pareil contexte.

\section{MÉTHODOLOGIE}

Entre septembre 2012 et janvier 2013 (c.-à.-d. avant la modification des lois canadiennes sur la fin de vie), 11 infirmières ont été interviewées dans le cadre de cette étude qualitative. Les participantes ont été recrutées dans divers services de soins d'un même établissement en centre urbain du Québec, soit un grand hôpital universitaire affilié doté d'un programme en oncologie reconnu. Ce programme en oncologie possède une unité spécialisée en soins palliatifs. Comme nous voulions obtenir une perspective infirmière d'ensemble, nous avons sciemment ciblé et retenu des infirmières spécialisées et non spécialisées en soins palliatifs. Six infirmières $(55 \%)$ travaillaient à l'unité spécialisée en soins palliatifs et y accomplissaient diverses fonctions de soins directs aux patients ou de consultation. Les cinq autres infirmières en oncologie (45\%) soignaient les cas aigus du programme d'oncologie répartis sur deux étages ou vus en consultation externe. Même si leur expérience variait, la plupart des infirmières de l'étude cumulaient entre 5 et 10 années d'expérience. Huit des infirmières interviewées détenaient un diplôme universitaire (72\%), parmi lesquelles quatre (36\%) détenaient un diplôme d'études supérieures.

Menées par deux des auteurs, les entrevues ont duré de 30 à 60 minutes. Elles ont été textuellement retranscrites pour l'analyse. La collecte des données et l'analyse ont eu lieu simultanément, et les premières entrevues ont permis la création de questions supplémentaires pour les entrevues suivantes. On a demandé précisément aux participantes comment le désir de mourir se manifestait et devenait une préoccupation chez un patient ayant un cancer avancé, quels sont les défis auxquels elles devaient faire face, comment elles réagissaient lorsqu'elles suspectaient qu'un patient envisageait la mort (quels étaient les sentiments, pensées, inquiétudes, paroles et gestes des participantes), qu'est-ce qui était à la base du désir de mourir d'un patient en phase terminale, et comment elles soignaient un patient ayant exprimé un désir de mort.

Une analyse systématique du contenu des entrevues a permis de relever les principaux thèmes et tendances dans les données (Lincoln et Guba, 1985). Deux des auteurs ont lu et relu chaque transcription pour se plonger dans le récit des participantes. Des procédures de codage ouvert, puis ciblé, ont permis d'élaborer une liste par catégories faisant ressortir les principales idées contenues dans le texte des entrevues. Après la première ronde d'analyse et la production des résultats préliminaires, deux autres auteurs ont relu les transcriptions d'entrevues et ont vérifié la précision des interprétations par rapport aux données originales, afin de valider les analyses préliminaires et la création des nouvelles perspectives. Durant le processus analytique, les auteurs se sont tous régulièrement rencontrés pour discuter du contenu des entrevues et pour élaborer, remettre en question et raffiner les interprétations en cours. 


\section{CONSTATS}

Dans les sections suivantes, nous répondons à notre question de recherche sur l'engagement des infirmières dans un contexte de désir de mort en décrivant trois thèmes tirés des entrevues. Les deux premiers thèmes décrivent l'engagement des infirmières en oncologie en tant que processus d'interprétation (thème 1) et l'application de la présence thérapeutique (thème 2). Nous soulignons ensuite les éléments manifestes dans les récits des infirmières qui semblent entraver la capacité d'engagement auprès des patients qui envisagent la mort (thème 3 ).

\section{Thème 1 : Interprétation du désir de mort chez un patient}

Je crois qu'il faut faire attention à ce que l'on entend par ce mot; le fait qu'ils disent vouloir mourir ne signifie pas toujours qu'ils veulent mourir.

Nous avons découvert que, chez nos participantes, le premier aspect de l'engagement auprès des patients qui envisagent la mort en est un d'interprétation. Les infirmières ont précisé chercher à comprendre ce qui est important pour le patient en portant attention aux signes verbaux, non verbaux et comportementaux. Les infirmières semblent se mettre au diapason du vécu qui entoure le désir apparent de mort du patient, cherchant ainsi à mieux comprendre ce qu'un tel désir peut réellement signifier pour le patient visé. Dans cette citation, une infirmière décrit le désir de mort exprimé comme une invitation, soit une « ouverture » au dialogue et à l'exploration entre l'infirmière et le patient.

[...] les patients le disent, et c'est une possibilité pour questionner et parler de n'importe quoi parce que quelque chose de très important vient d'être dit. C'est la dernière partie de leur vie [...] ils vous disent ce qu'ils veulent, puis les portes s'ouvrent sur l'exploration [...] et lorsque cela se produit, je dépose mes choses, je m'assois et je saisis l'occasion [...]

En tirant profit de « l'ouverture » que les patients fournissent, une invitation à mieux comprendre le vécu vers la mort, les infirmières ont décrit comprendre de manière nuancée les divers sens attribuables au désir de mort des patients. Il peut s'agir d'une perte d'enthousiasme pour continuer de vivre, du désir d'avoir de meilleurs soins infirmiers et de reprendre le contrôle de sa vie ou d'une frustration liée à une situation sans issue. Les infirmières ont aussi souligné que la nature du désir de mort d'un patient peut fluctuer avec le temps. Il n'y a donc pas, dans le témoignage des infirmières, de signification uniforme du désir de mort applicable dans leur pratique, parce qu'une interprétation attentive du désir de mort d'un patient peut potentiellement révéler un chevauchement de « désirs » contradictoires.

Eh bien, il [le désir de mort] peut signifier plusieurs choses, et cela en fait un concept épineux selon moi [...] Je crois que parfois les gens veulent cesser de souffrir, ou veulent éviter de souffrir davantage, de perdre leur dignité, leur indépendance, alors ce que pense le patient s'emmêle. Cela peut vouloir signifier différentes choses selon le patient.

Chez certaines participantes de l'étude, il y avait une tendance à accorder différentes significations au désir de mort de leurs patients plutôt qu'à y voir un réel désir de mourir.
[...] il y a quelques années, j'ai soigné un homme dans la jeune cinquantaine atteint d'un cancer de l'estomac [...] durant les deux à quatre derniers mois de vie, il n'avait que la peau et les os [...] il ne pouvait plus jouer au hockey, il vivait beaucoup de pertes [...] Je crois qu'il ne savait plus trop ce qui le définissait comme personne. Il ne se ressemblait plus, se sentait toujours mal, ne voulait plus manger, mais avait peur de mourir de faim s'il ne mangeait plus [...] il avait peur de ne plus être là pour sa famille [...] il disait : "Je suis un fardeau pour tout le monde et je ne me sens plus du tout moi-même, je ne sais pas si je peux continuer ainsi. Je meurs de faim et je veux seulement mourir. " Il ne disait pas qu'il voulait vraiment le faire, ou qu'il voulait que nous le fassions, mais il traversait cette difficile période de questionnement : «À quoi ça sert?», «Comment puis-je améliorer les choses?»

Pour cette infirmière, la signification du désir de mort de son patient n'est pas vue comme un recours à l'aide à mourir, c'est-à-dire un recours à l'euthanasie ou au suicide assisté, mais elle est plutôt une réaction à l'expérience de diverses pertes à une phase avancée de la maladie. En fait, le patient y est décrit comme vivant des émotions perturbantes typiques en quelque sorte du vécu de fin de vie, dont l'impression de perdre son individualité et d'être un fardeau pour autrui. Plus tard dans la même entrevue, l'infirmière ajoute une réflexion sur son patient :

[...] C'est délicat, il n'a pas dit « Je veux mourir » ou «Je veux mettre fin à cela », il a plutôt tourné autour du pot. Ses propos étaient du genre: "Je ne sais pas si je peux continuer à vivre ainsi et je ne sais pas combien de temps encore je peux faire cela».

Cette infirmière a sciemment choisi de parler de ce patient en particulier. Il ne s'agissait pas d'un patient qu'elle venait de soigner, et il ne lui paraissait pas transmettre explicitement un message clair et cohérent sur un désir de mort. Les contradictions dans son récit (« je veux seulement mourir », il n'a pas dit « je veux mourir ») démontrent à quel point les impressions des infirmières sur le vécu de leurs patients résultent d'un processus interprétatif. Dans le passage ci-après, l'infirmière établit une distinction entre soigner quelqu'un qui recherche une « aide » pour hâter sa mort, et soigner un patient qui recherche quelqu'un à qui confier son vécu émotif.

[...] ils $y$ ont fait allusion sans se montrer, disons, directs : "J'en ai assez », "Je suis fatigué », "Je veux juste partir », vous voyez ce que je veux dire. Et dans ces cas, je n'ai pas vu ou senti qu'ils demandaient mon aide ou qu'ils voulaient autre chose que d'exprimer leurs sentiments.

Dans la réflexion ci-dessus, on ne voit pas clairement comment et pourquoi la participante « sentait » (c.-à-d. interprétait) que le désir du patient était un accompagnement relationnel (avoir une oreille attentive à son écoute) et non l'obtention d'une « aide » directe pour mourir.

\section{Thème 2 : Offrir une présence thérapeutique}

[...] ils nous envahissent en quelque sorte, mais pas de manière négative [...] C'est un peu comme s'ils nous disaient de ne pas les laisser seuls. 
Nous avons découvert que la présence thérapeutique est une valeur fondamentale essentielle pour les infirmières de notre étude. Nos participantes ont décrit cette nécessité d'être à la « disposition » des patients et se voient comme un « accompagnateur discret ». Ce que signifie cette présence cependant n'était pas chose facile à expliquer pour les infirmières. Dans cette section, nous décrivons les éléments tirés des réflexions des participantes portant directement sur leurs réactions à l'égard des patients, pour mettre davantage en lumière le sens donné au mot engagement dans un contexte de soins aux patients qui envisagent la mort.

Les infirmières ont réfléchi aux difficultés associées au fait d'être « simplement présentes » dans ces cas, vu l'absence de solutions évidentes et de réponses faciles. Les hésitations et insécurités des infirmières durant les conversations difficiles sur le désir de mort et sur la fin de vie en général pouvaient interférer avec l'engagement, notamment lorsqu'elles craignaient que ces conservations blessent par inadvertance le patient.

Je me sens mal d'en parler. Et s'ils ne désirent pas mourir, que vont-ils penser de moi qui leur pose la question? On a peur de leur réaction, vous savez; ils pourraient penser : "Est-ce que ça signifie qu'elle pense que je vais mourir? »C'est donc un sujet délicat. Honnêtement, je ne suis pas la plus à l'aise avec le sujet.

Les infirmières acceptent aussi difficilement les limites de leurs aptitudes à contrôler, régler et offrir quelque chose de concret et de tangible pour calmer la souffrance de leurs patients.

J'imagine qu'une infirmière se sent toujours déchirée [...] car on essaie toujours de régler tout. On essaie toujours de tout améliorer et là, on ne peut rien améliorer; le patient ne peut pas se sentir mieux et on ne peut pas vraiment lui faciliter la mort, alors c'est difficile.

Même si la présence a été largement vue comme essentielle dans le cadre des soins aux patients en fin de vie, bien des infirmières ont décrit leur questionnement sur l'efficacité de leur présence lorsque la souffrance est intense. Elles ont même douté de leurs propres capacités et aptitudes comme infirmières. Fait intéressant, si certaines doutaient d'elles-mêmes dans un contexte de désir de mort, d'autres ont exprimé un doute semblable dans un contexte de discussion plus large de transition entre les soins curatifs et les soins palliatifs. Le contenu des réflexions, dans chaque cas, était remarquablement similaire. À titre d'exemple, voici les réflexions de deux participantes; la première porte sur le désir de mort, et la seconde, sur les soins palliatifs :

[...] ce sentiment d'échec, quand ils demandent à mourir, vient-il de ce que vous n'avez pas su gérer adéquatement leur confort, faire plus pour eux ou pu répondre à leurs attentes sur les soins? [...] Est-ce pour cela qu'ils demandent maintenant de mourir?

[...] Je crois que beaucoup de mes collègues médecins et infirmières, lorsque vient le moment de songer aux soins palliatifs, ont le sentiment d'avoir échoué [...] On a limpression de ne pas avoir tout fourni et de ne pas avoir fait du mieux que l'on pouvait [...]
Dans les deux cas, le risque de se sentir en échec est évident. Par conséquent, il semble que le désir de mort représente chez ces infirmières un contexte où se manifeste un défi plus fondamental sur les soins en fin de vie : celui d'accepter les limites humaines à régler les souffrances d'autrui. Dans les passages suivants, nous voyons une infirmière vivre ce défi :

[...] même l'infirmière la plus expérimentée peut se sentir submergée dans un tel cas... C'est en parlant à mes collègues que j'ai pu normaliser le tout... C'est bon et rassurant de savoir qu'il ne s'agit pas de mon savoir, mais bien d'un cas légitimement difficile, et que toutes, même les infirmières en oncologie plus expérimentées, ont vécu cette situation qui s'avère en réalité difficile pour n'importe qui, expérience ou pas...

Cette participante, en utilisant les mots " cas légitimement difficile » et en comparant son expérience à celle « d'infirmières en oncologie plus expérimentées », semble valider que les difficultés auxquelles elle fait face, c'est-àdire de ne pas pouvoir répondre à la souffrance d'autrui, sont inhérentes à la situation de soins; il ne s'agit pas du résultat d'un manque d'expérience ou d'incompétence. C'est dans ce contexte obligeant à délaisser l'approche de soins de fin de vie consistant à tenter de tout régler que la notion d'être « simplement présente » devient pertinente et émerge.

J'ai appris au fil des ans qu'il faut parfois mettre de côté ce désir (d'agir). C'est apprendre durant ces moments où l'on est avec l'autre, durant les moments les plus difficiles où l'on est simplement présent... c'est apprendre à être calme et confortable, à être calme et à écouter plutôt que de chercher à s'occuper en faisant quelque chose qui NOUS fait sentir à l'aise.

À la fin de l'entrevue, on a demandé à une infirmière en quoi travailler avec des patients voulant mourir s'est avéré bénéfique dans sa pratique. Elle a répondu être devenue « plus authentique » parce qu'elle avait « laissé tomber le sentiment d'avoir pour responsabilité de tout régler pour eux ». Nous avons aussi trouvé dans nos données que le désir de mort invitait l'infirmière à un meilleur engagement relationnel dans l'expérience de fin de vie du patient :

" [...] Cela favorise une relation de confiance. Le patient se montre honnête avec moi sur ses sentiments et c'est important [...] lorsque quelqu'un vous dit vouloir mourir, cela peut signifier qu'il a un manque à combler : un besoin de soutien psychologique, de prise en charge des symptômes, de ce que l'on a à notre portée. Mais je crois que lorsqu'un patient nous exprime un sentiment aussi important, notre niveau d'engagement s'intensifie ou augmente parce qu'on veut les aider à travers ça. »

Dans le passage précédent, la participante décrit entendre dans le désir de mort un « indice » quant aux besoins du patient. Cette description est cohérente avec l'idée selon laquelle l'engagement pose un défi d'interprétation, comme décrit précédemment. Dans cette réflexion, cependant, la description de l'infirmière passe de l'interprétation à l'interaction. Elle mise sur le fait qu'elle peut avoir la chance de gagner et d'approfondir la confiance du patient, en haussant volontairement son « niveau d'engagement ». Ce qui transparaît aussi dans la réflexion de l'infirmière, c'est le caractère délicat des implications morales liées à un tel attachement; elle respecte l'ampleur de ce qu'on partage avec elle, ce qui l'amène à réagir. 


\section{Thème 3 : Forces entravant l'engagement}

[...] Il y a des jours où l'on n'arrive pas à faire ce que l'on devrait faire [...]

Dans les sections précédentes, nous avons montré que l'engagement infirmier auprès des patients envisageant la mort est un processus d'interprétation et de présence. L'interprétation est caractérisée par l'ouverture de l'infirmière à une série de significations recouvrant un « désir de mort » qui, pour être mise au jour, exige une lecture attentive et comprend parfois des signes très subtils. La présence entre en jeu lorsque les infirmières reconnaissent une occasion créée par le désir de mort et agissent en conséquence en vue de renforcer leur lien thérapeutique avec le patient.

S'engager auprès des patients qui envisagent la mort, tel que décrit précédemment, présente toutefois des défis. Dans cette section, nous expliquons les forces qui interfèrent avec l'engagement relationnel lorsque les patients désirent mourir. La première de ces forces est temporelle. Toutes les infirmières ont mentionné le manque de temps comme facteur gênant leur aptitude à s'engager auprès des patients et à leur fournir les soins attentifs qu'un désir de mort exige. Les deux réflexions suivantes l'illustrent bien :

[...] Il y a sur l'étage en ce moment un patient très agité, qui m'a dit: «Je vous en prie, tuez-moi ». Et il veut que je reste là pour lui tenir la main, ce qui est facile à faire, mais j'ai aussi trois ou quatre autres patients, ou plus, et je ne peux pas [...] Veut-il mourir parce qu'il veut mourir ou veut-il mourir parce que personne ne s'assoit avec lui pour lui tenir la main?

Parce qu'il est certain qu'un patient qui veut mourir nous ramène dans la réalité des soins infirmiers, et cette réalité est que nous ne pouvons donner tant de temps et d'attention à quelqu'un qui veut mourir par rapport à ce que nous donnerons à quelqu'un de gravement malade mais qui désire vivre [...] On n'a tout simplement pas le temps; ce n'est pas parce qu'on ne veut pas, c'est qu'on n'a pas le temps de régler la situation de la même façon.

La seconde force est législative. Au moment de mener cette étude, l'euthanasie et le suicide assisté étaient illégaux au Canada et au Québec, et ils ne faisaient pas partie de la pratique infirmière. En discutant des soins aux patients envisageant la mort, les infirmières semblaient préférer discuter des patients dont le désir de mort représentait un sentiment de détresse généralisée plutôt qu'une requête délibérée de hâter leur mort. Pour l'une des participantes, l'idée de vouloir hâter la mort ne s'était jamais présentée à elle et elle la considérait comme « hors du champ » de sa pratique. Durant son entrevue, le désir de mort n’a jamais été perçu comme un désir de mort délibéré.

[...] Mais nous n'avons eu aucun cas où la personne a demandé à hâter sa mort. Il y a eu des personnes qui ont désiré la mort ou qui étaient épuisées et qui disaient : «Ça suffit, je veux juste mourir » [...], mais personne n'a dit «SVP mettez fin à ma vie » ou « je vais mettre fin à ma vie ». Lorsque cela survient, la situation est très différente. C'est à l'extérieur du champ d'intervention de notre équipe [...] Je parle davantage de gens qui ont perdu le désir de vivre.
Pour d'autres participantes, toutefois, c'était une réalité dans leur pratique de soigner au quotidien des patients ayant exprimé (à l'infirmière) un désir de mort pour la hâter.

[...] les patients voulant vraiment être tués, ce qui est contraire à l'éthique, et qui demandent constamment plus de morphine, ou certains patients qui ont des gouttes souscutanées pour la douleur et qui essaient d'ajuster la dose de la pompe, ce qui présente une difficulté.

Il convient de noter le commentaire « contraire à l'éthique » dans l'évaluation de la réponse à une demande visant à hâter la mort. Il réfère au contexte législatif qui a servi à limiter la cible et le contenu des récits de nos participantes. Ainsi, les participantes n'ont formulé que très peu de commentaires sur l'engagement auprès de patients dans un contexte de désir de mort réel. Il est possible que pour ces infirmières, l'euthanasie et le suicide assisté constituaient des pratiques si étrangères qu'elles y étaient indifférentes ou incapables d'en parler en détail. Ce qui ressort dans les données, toutefois, ce sont les descriptions sur les limites à l'engagement. Les infirmières étaient manifestement limitées dans leur capacité à fournir une présence thérapeutique lorsque les patients voulaient hâter leur mort; dans ces cas, on demandait aux infirmières de faire quelque chose qui leur était tout simplement impossible.

Que leur dit-on, comment leur fait-on comprendre ce qu'on peut faire pour eux, qu'ils nous importent [...] ils ne veulent qu'une seule chose de vous, et c'est de hâter leur mort, et c'est une difficulté.

J'éprouvais une grande aversion lorsqu'il me le demandait, quand il voulait en parler [...] C'est au point que je ne voulais plus lui parler autrement que pour lui dire que j'avais bien entendu et que je savais qu'il vivait une situation difficile, et que je n'avais aucune réponse à lui fournir. Il pleurait. J'étais capable de lui fournir un peu de réconfort, vous savez, mais après il cherchait à recommencer à en parler et je prenais cela très négativement.

Si peu de participantes ont parlé de demandes explicites d'euthanasie et de suicide assisté, la réflexion des infirmières interrogées sur leur vécu avec des patients voulant la mort s'est plutôt tournée vers les patients ayant choisi de mettre fin au traitement prolongeant la vie. Pour bien des infirmières, mettre fin au traitement était vu comme un résultat naturel chez une personne voulant la mort et considéré par toutes comme un choix de fin de vie acceptable. Les réflexions des infirmières sur l'arrêt du traitement dans un contexte de désir de mort tournaient beaucoup autour des conflits familiaux, et les infirmières ont dit qu'elles trouvaient difficile de concilier le respect de l'autonomie du patient et ce que souhaitait la famille. Dans ces circonstances, les infirmières ont décrit que le soutien au patient pouvait restreindre leur capacité à être entièrement présentes auprès de la famille.

Je pense que la famille appréciait ma présence, mais elle avait beaucoup de difficulté à accepter que nous soutenions le patient dans sa décision de mourir. Je pense qu'on nous voyait comme des professionnelles de la santé supposées tout faire en notre pouvoir pour sauver quelqu'un, alors elle comprenait difficilement que nous laissions leur père s'en aller. 


\section{DISCUSSION}

Comme l'aide médicale à mourir vient tout juste d'être légalisée au Canada, toutes les infirmières devront réfléchir à leur expérience acquise auprès de patients envisageant la mort et voir comment elles pourront désormais la mettre en pratique dans les soins aux patients qui demanderont et recevront l'aide à mourir. Selon des statistiques colligées dans les pays où l'aide à mourir est légalement pratiquée, la plupart des demandes pour hâter la mort sont formulées dans un contexte de diagnostics de cancer graves et en phase terminale (OnwuteakaPhilipsen et al., 2012; Oregon Public Health Division, 2013). Par conséquent, même si nos résultats s'appliquent à toute infirmière soignant des patients en fin de vie, ils sont particulièrement pertinents pour les infirmières en oncologie. Dans cette section, nous identifions certaines implications tirées de nos constats pour amorcer une réflexion, chez les infirmières en oncologie, sur l'engagement thérapeutique auprès des patients qui envisagent la mort et reçoivent des soins de fin de vie à l'intérieur du nouveau cadre législatif canadien.

Même si le sujet central de nos entrevues portait sur les soins aux patients désirant mourir, les participantes ont souvent dévié du sujet et parlé de manière générale des soins de fin de vie. Nous avons par conséquent découvert, à notre surprise, qu'inviter des infirmières à parler du désir de mort n'a pas principalement donné lieu à des conversations sur la formulation de demandes d'euthanasie ou de suicide assisté. Nos participantes ont en général parlé de la complexité, des obstacles et des possibilités en jeu dans les soins de fin de vie offerts aux patients. Elles ont très souvent énoncé leur point de vue sur le désir de mourir à l'intérieur de plus grands discours sur la fin de vie, notamment sur la suspension ou l'arrêt des soins prolongeant la vie, et sur la tension entre les approches curatives et palliatives. Chez ces participantes, l'idée même du désir de mort n'était pas exceptionnelle, controversée ou choquante. De fait, bon nombre de participantes semblaient trouver normale l'idée qu'on puisse désirer mourir dans un contexte de maladie en phase terminale.

Les définitions cliniques et légales de mort assistée sont relativement uniformes en contexte international. Pour éviter toute ambiguïté terminologique, ces définitions réduisent méticuleusement l'euthanasie à ses éléments constituants. Les définitions standards cherchent à établir clairement les limites conceptuelles et empêchent toute confusion entre l'aide à mourir et d'autres pratiques médicales de fin de vie. En voici un exemple : «Aux Pays-Bas, l'euthanasie est définie depuis 1985 comme l'administration de médicaments avec l'intention explicite de mettre fin à la vie à la demande explicite d'un patient » (Rietjens, van der Maas, Onwuteaka-Philipsen, van Delden et van der Heide, 2009, p. 272, traduction, nous soulignons). Le risque avec de telles définitions est qu'elles décrivent l'aide à mourir comme un acte ponctuel circonscrit dans un cadre relativement restreint dans l'espace et le temps, c'est-à-dire le moment où les médicaments sont administrés et où la mort par euthanasie survient. La compréhension anthropologique contemporaine de l'aide à mourir, toutefois, problématise l'interprétation simpliste de cette pratique comme acte ponctuel. À titre d'exemple, dans une étude ethnographique sur l'euthanasie aux Pays-Bas, Norwood (2007) a trouvé que l'euthanasie est un processus communicatif, un ensemble d'échanges entre les patients et les médecins, qui peut connaître à de multiples reprises des pauses et de nouveaux départs. Lorsqu'on y voit un processus de soins relationnel se dessinant dans le temps, « l'euthanasie » ne mène pas nécessairement à la mort des patients, ces derniers ayant pu retirer leur demande ou mourir avant de la mener à bien. Une conceptualisation plus large alors de l'aide à mourir, comme processus de soins relationnel, inclut les nombreux moments qui surviennent entre les patients, les membres de la famille et les professionnels de la santé, à partir du moment où les patients suggèrent que l'euthanasie est quelque chose qu'ils envisagent, jusqu'au moment où ils meurent (par euthanasie ou autrement) ou statuent que l'euthanasie n'est finalement pas pour eux. Ces moments exigent qu'il faille répondre aux incertitudes et aux peurs des patients, les aider à comprendre et à explorer leurs choix, et faciliter le dialogue entre eux et leurs proches. Conceptualiser l'aide à mourir plus largement, comme un processus de soins dialogique qui survient dans une relation thérapeutique, signifie que des étapes précises et spécifiées dans une loi pertinente (évaluation des compétences, consultation auprès d'un deuxième médecin, administration de médicaments) ne sont pas du tout suffisantes pour décrire la complexité et les nuances inhérentes à la réalité et à la réalisation vécues de la mort assistée à titre de pratique thérapeutique.

Le problème de savoir quelle infirmière administrera ou non les médicaments mortels dans l'aide à mourir, tel que décrit au début de cet article, passe en quelque sorte à côté de la notion de participation des infirmières dans les processus de soins associés à l'aide à mourir. Une analyse sur les infirmières et l'aide à mourir analysée récemment pour être publié dans la littérature internationale montre que le vécu des infirmières impliquées dans l'euthanasie ou le suicide assisté est caractérisé par un fort sentiment de responsabilité morale (Elmore, Wright et Paradis, 2016). En raison de leurs étroites interactions avec les patients, les infirmières sont souvent les premières professionnelles de la santé à entendre les demandes d'aide à mourir. Ces demandes viennent ébranler les infirmières sur le plan éthique et émotionnel, qui veillent alors à ce que les patients qui formulent ces demandes reçoivent les meilleurs soins possible, par exemple, en mesurant ce que cette demande peut cacher d'autre, en trouvant des solutions palliatives ou en défendant la cause du patient auprès du médecin pour que sa demande soit prise au sérieux. Des facteurs contextuels, comme le temps requis pour créer des liens thérapeutiques avec les patients et la qualité de la collaboration interprofessionnelle viennent grandement influencer la prise de conscience sur les responsabilités qu'ont les infirmières soignant des patients en fin de vie (De Bal et al. 2006; Denier et al., 2009; Denier, Dierckx de Casterle et al. 2010; Denier, Gastmans et al., 2010; Dierckx de Casterle et al., 2010; Dierckx de Casterle, Verpoort, De Bal et Gastmans, 2006; Harvath et al., 2006; Matzo et Schwarz, 2001; Schwarz, 2003, 2004; Volker, 2001, 2003). Par conséquent, le vécu des infirmières devant l'aide à mourir constitue une expérience morale 
où l'engagement de l'infirmière dans la mort assistée se répercutera dans les valeurs qu'elle juge importantes (Beagan et Ells, 2009; Hunt et Carnevale, 2011).

Dans cette étude, qui a été menée auprès d'infirmières avant que les lois canadiennes permettent l'euthanasie et le suicide assisté comme pratique de soins de fin de vie admissible, les infirmières ont parlé des façons dont elles s'engagent auprès des patients en phase terminale qui envisagent la mort. Il s'agit de patients qui, sous les nouveaux régimes et ceux à venir, pourront potentiellement accéder à des avenues de soins offrant l'euthanasie et le suicide assisté, alors il est pressant de regarder attentivement ce que signifie l'idée d'engagement thérapeutique avec ces patients dans la pratique des infirmières en oncologie. D'abord, nos constats soulignent l'importance de reconnaître dans la pratique infirmière clinique la nature complexe d'une demande visant à hâter la mort. Selon Mak et Elwynn (2005), « [v]ouloir l'euthanasie ne peut être interprété exclusivement au premier degré. Le sens de cette demande ne se confine pas à la réalité de la désintégration physique ou de la souffrance entraînée par les effets du cancer, mais cache des peurs et des préoccupations existentielles concernant le désir d'établir des liens, d'être soigné et d'être respecté, le tout compris dans un contexte de vécu holistique du patient » (p. 348, traduction, nous soulignons). Le défi pour les infirmières qui soignent des patients envisageant la mort est de s'ouvrir à l'éventail de significations sous-jacentes au désir de mort et d'utiliser leurs connaissances sur ces significations pour moduler leurs réactions (Gudat, 2015; Houtepen et Hendrikx, 2003; Müller-Busch, 2015; Ohnsorge, Gudat Keller, Widdershoven, et Rehmann-Sutter, 2012).

Selon Ann Gallagher, éminente infirmière éthicienne, « une pratique infirmière exemplaire n'est pas seulement minutieuse, mais aussi fragile parce que les liens sont négociés et soutenus dans des contextes d'incertitude » (Gallagher, 2012, p. 712, traduction). Dans un contexte d'aide à mourir, les infirmières doivent reconnaître l'incertitude inhérente à l'engagement auprès d'un patient qui manifeste un désir de mort. L'éthique relationnelle en soins infirmiers est une perspective qui nous amène à considérer la qualité des relations humaines comme point central de la recherche infirmière, en reconnaissant que l'engagement entre l'infirmière et le patient est un élément fondamental de ce que signifient offrir et vivre des soins infirmiers de qualité en fin de vie (Davies et Oberle, 1990; Mok et Chiu, 2004; Wright et al., 2009). L'engagement dans ce contexte signifie que les infirmières « découvrent l'engagement moral du lien et y réagissent » (Bergum, 2013, p. 135-136, traduction), ce qui dans les soins de fin de vie aux patients atteints de cancer implique un engagement à être présent pour le patient et sa famille (Barnard et al., 2006; Wright et Pugnaire Gros, 2012). Comme l'a observé Cramer (2002) : « Parfois le simple fait "d'être là" est ce qui importe le plus. Nous appelons cela "le pouvoir de la présence”. Il n'est pas nécessaire d'avoir les “bons mots" ou de dire les "bonnes choses" auprès des patients, mais juste d'être là comme témoin, de leur tenir la main, de montrer qu'on prend soin d'eux; ça peut donner autant que n'importe quelle intervention hypertechnologique d'avant-garde qu'on peut offrir. » (p. 55, traduction).
Naturellement, la présence thérapeutique dans les soins de fin de vie, y compris dans les soins des patients voulant la mort, n'est pas une activité insignifiante. Elle demande de l'attention, de l'engagement et des habiletés (Strang, Henoch, Danielson, Browall et Melin-Johansson, 2014). Si nos participantes ont parlé avec éloquence de l'éventail de significations sous-jacentes au désir de mort et des processus de présence sophistiqués requis pour s'engager de manière appropriée face à ces significations, elles ont aussi parlé des forces contextuelles qui entravent leurs aptitudes à amorcer de manière appropriée leur engagement. Selon Dierckx de Casterle (2015), le rôle de l'infirmière comme accompagnatrice compétente est mis en danger par les environnements de soins « très techniques, rationnels, fragmentés et plutôt chaotiques » (p. 3332, traduction), car ceux-ci n'accordent pas suffisamment de valeur aux capacités d'engagement relationnel des infirmières à titre de pratique éthique essentielle, mais aussi par les infirmières elles-mêmes lorsqu'elles adoptent en réaction une pratique éthique conformiste, c'est-à-dire une pratique exigeant que les infirmières suivent les conventions (ordonnances médicales, règles et normes d'une unité de soins, procédures et directives) sans réfléchir de manière créative et critique sur ce qui est « bien ». Les conventions « doivent faire l'objet d'une évaluation critique en contexte et en termes de contribution au mieux-être des patients » (Dierckx de Casterle, Izumi, Goetfrey et Denhaerynck, 2008, p. 547, traduction). Lorsque les patients envisagent ou demandent l'aide à mourir, il faut qu'il y ait, de la part de tous les professionnels de la santé, y compris les infirmières, une pratique songée et profondément réfléchie. La façon pour les infirmières d'interpréter et de réagir à leurs patients, dans une pratique relationnelle facilitée ou limitée en contexte de travail, influencera fortement leur aptitude à fournir des soins éthiques dans le nouveau paysage législatif de soins de fin de vie au Canada.

\section{LIMITES ET PROCHAINES ÉTAPES}

Cette étude qualitative a été menée auprès de 11 infirmières travaillant dans divers secteurs du programme d'oncologie d'un hôpital universitaire bien établi en secteur urbain. Dans d'autres milieux de soins, comme en soins communautaires, la façon dont les infirmières décriraient leur engagement auprès des patients qui veulent mourir pourrait être influencée de manières que nous n'avons pu cerner dans cette étude. Pour cette raison, de futures études auprès d'infirmières travaillant dans divers contextes où des Canadiens reçoivent des soins de fin de vie seraient précieuses. De plus, même si notre échantillon contenait des infirmières provenant de l'intérieur et de l'extérieur de l'unité spécialisée en soins palliatifs (toutes dans un même programme d'oncologie), nous n'avons pu identifier de tendances cohérentes ni de différences particulières dans les descriptions des infirmières, qu'elles aient eu ou non une expertise en soins palliatifs. De plus, nous n'avons pas demandé aux participantes de révéler leurs orientations religieuses ou culturelles, qui pourraient influencer leur attitude par rapport à l'aide à mourir. Il pourrait s'avérer intéressant de mener une future recherche sur l'incidence de la religiosité ou d'autres sources d'influence culturelle sur l'engagement 
des infirmières auprès des patients qui veulent mourir. Enfin, même si cette étude par entrevues donne un aperçu de la façon dont les infirmières discutent de l'engagement thérapeutique avec les patients voulant mourir, et de leurs réflexions, il serait profitable dans une future recherche d'utiliser l'observation des participantes comme méthode pour apporter un éclairage sur la façon dont les infirmières amorcent leur présence thérapeutique dans un contexte de désir de mort. Des études ethnographiques sur la pratique des infirmières en soins de fin de vie dans le nouveau cadre législatif canadien permettraient, par exemple, de déterminer les défis comme les possibilités liés à l'éthique de soins auprès des patients demandant l'aide à mourir, ainsi que de clarifier le rôle de l'infirmière dans ce processus.

\section{CONCLUSION}

Cette étude, qui a été menée avant les changements législatifs, démontre comment un contexte législatif où l'aide à mourir est prohibée peut restreindre l'engagement thérapeutique. En effet, les infirmières ne pouvaient réagir lorsqu'elles avaient l'impression que leurs patients leur posaient des questions auxquelles elles ne pouvaient répondre. Ces résultats reflètent le sentiment d'impuissance que des chercheurs belges ont décrit dans leur étude sur le vécu des infirmières avant la légalisation de l'euthanasie dans ce pays (De Bal et al., 2006). Maintenant que l'aide à mourir est légale au Québec, et qu'elle pourrait bientôt l'être au Canada selon létat des lieux au moment d'écrire ces lignes, les infirmières

\section{RÉFÉRENCES}

Association des infirmières et infirmiers du Canada (AIIC/CNA). (2015). Sommaire à lintention du Comité externe sur les options de réponse législative à Carter c. Canada (2015) établi par le gouvernement $d u$ Canada. Consulté sur https://www.cna-aiic. ca/ /media/cna/page-content/pdf-fr/aide-medicale-a-mourir_ sommaire-a-lintention-du-comite-externe-sur-les-options-dereponse-legislative-a-carter-c-canada.pdf?la=fr

Austin, W.J. (2011). The incommensurability of nursing as a practice and the customer service model: An evolutionary threat to the discipline. Nursing Philosophy, 12, 158-166.

Barnard, A., Hollingum, C. et Hartfiel, B. (2006). Going on a journey: Understanding palliative care nursing. International Journal of Palliative Care, 12(1), 6-12.

Beagan, B. et Ells, C. (2009). Values that matter, barriers that interfere: The struggle of Canadian nurses to enact their values. Canadian Journal of Nursing Research, 41(1), 86-107.

Bergum, V. (2004). Relational ethics in nursing. Dans J.L. Storch, P.A. Rodney et R.C. Starzomski (Éd.), Toward a moral horizon: Nursing ethics for leadership and practice. Toronto (Ont.) : Pearson Canada Inc. 485-503.

Bergum, V. (2013). Relational ethics for health care. Dans J.L. Storch, P.A. Rodney et R.C. Starzomski (Éd.), Toward a moral horizon: Nursing ethics for leadership and practice. Toronto (Ont.) : Pearson. 127-142.

Carter c. Canada (2015). Procureur général, 2015 CSC 5.

Cramer, C.F. (2002). To live until you die: Quality of life at the end of life. Clinical Journal of Oncology Nursing, 14(1), 53-56.

Cronqvist, A., Theorell, T., Burns, T. et Lutzen, K. (2004). Caring about - caring for: Moral obligations and work responsibilities in intensive care nursing. Nursing Ethics, 11(1), 63-76. en oncologie pourront en théorie ne plus se sentir contraintes par un contexte légal restreignant. Elles devraient maintenant être en mesure de discuter ouvertement et honnêtement avec leurs patients d'un spectre plus grand de choix de fin de vie. Par ailleurs, les infirmières doivent continuer de s'opposer à une approche « de résolution rapide » de la souffrance de leurs patients, ce qui, selon nos constats, est une lutte constante pour les infirmières en oncologie qui soignent des patients en fin de vie. Les habiletés d'interprétation et de présence en soins infirmiers seront plus que jamais pertinentes, et les patients qui exprimeront un désir de mort dans le cadre du nouveau régime législatif auront besoin d'un engagement thérapeutique auront besoin d'un haut niveau d'engagement thérapeutique. Un désir de mort chez un patient atteint d'un cancer en phase terminale devrait envoyer le signal qu'il faut procéder à une analyse critique et réfléchie sur ce qui est sousjacent à la souffrance du patient, sur ce qui pourrait être fait pour l'aider, et sur l'optimisation potentielle des systèmes de prestation de soins de sorte que les infirmières puissent offrir au mieux les soins requis par le patient.

\section{FINANCEMENT}

David Kenneth Wright a reçu la Bourse de nouveau chercheur (2014-2017) de la Fondation de l'Hôpital St. Mary à Montréal. Cette recherche a aussi été financée par une subvention de recherche d'Axe sciences infirmières du Réseau québécois de recherche sur le suicide, financé par le Fonds de recherche du Québec-Santé (CP Wright et Bitzas).

Davies, B. et Oberle, K. (1990). Dimensions of the supportive role of the nurse in palliative care. Oncology Nurses Forum, 17(1), 87-94.

De Bal, N., Dierckx de Casterle, B., De Beer, T. et Gastmans, C. (2006). Involvement of nurses in caring for patients requesting euthanasia in Flanders (Belgium): A qualitative study. International Journal of Nursing Studies, 43(5), 589-599.

De Beer, T., Gastmans, C. et Dierckx de Casterle, B. (2004). Involvement of nurses in euthanasia: A review of the literature. Journal of Medical Ethics, 30(5), 494-498.

Denier, Y., Dierckx de Casterle, B., De Bal, N. et Gastmans, C. (2009). Involvement of nurses in the euthanasia care process in Flanders (Belgium): An exploration of two perspectives. Journal of Palliative Care, 25(4), 264-274.

Denier, Y., Dierckx de Casterle, B., De Bal, N. et Gastmans, C. (2010). «It's intense, you know.» Nurses' experiences in caring for patients requesting euthanasia. Medicine Health Care and Philosophy, 13(1), 41-48.

Denier, Y., Gastmans, C., De Bal, N. et Dierckx de Casterle, B. (2010). Communication in nursing care for patients requesting euthanasia: A qualitative study. Journal of Clinical Nursing, 19(2324), 3372-3380.

Dierckx de Casterle, B. (2015). Realising skilled companionship in nursing: A utopian idea or difficult challenge? Journal of Clinical Nursing, 24(21-22), 3327-3335.

Dierckx de Casterle, B., Denier, Y., De Bal, N. et Gastmans, C. (2010). Nursing care for patients requesting euthanasia in general hospitals in Flanders, Belgium. Journal of Advanced Nursing, 66(11), 2410-2420. 
Dierckx de Casterle, B., Izumi, S., Godfrey, N.S. et Denhaerynck, K. (2008). Nurses' responses to ethical dilemmas in nursing practice: Meta-analysis. Journal of Advanced Nursing, 63(6), 540-549.

Dierckx de Casterle, B., Verpoort, C., De Bal, N. et Gastmans, C. (2006). Nurses' views on their involvement in euthanasia: A qualitative study in Flanders (Belgium). Journal of Medical Ethics, 32(4), 187-192.

Doane, G.H. et Varcoe, C. (2007). Relational practice and nursing obligations. Advances in Nursing Science, 30(3), 192-205.

Elmore, J., Wright, D.K., \& Paradis, M. (2016, E-pub ahead of print). Nurses' moral experiences of assisted death. Nursing Ethics. DOI: 10.1177/0969733016679468.

Gallagher, A. (2012). Slow ethics for nursing practice. Nursing Ethics, 19(6), 711-713.

Government of Canada. (2016). An Act to amend the Criminal Code and to make related amendments to other Acts (medical assistance in dying) (S.C. 2016, c. 3). Retrieved from: http://laws-lois.justice. gc.ca/eng/AnnualStatutes/2016_3/

Gudat, H. (2015). From understanding to patient-centred management: Clinical pictures of a wish to die. Dans C. RehmannSutter, H. Gudat et K. Ohnsorge (Éd.), The Patient's Wish to Die: Research, Ethics, and Palliative Care. Oxford : Oxford University Press.

Harvath, T.A., Miller, L.L., Smith, K.A., Clark, L.D., Jackson, A. et Ganzini, L. (2006). Dilemmas encountered by hospice workers when patients wish to hasten death. Journal of Hospice and Palliative Nursing, 8(4), 200-209.

Houtepen, R. et Hendrikx, D. (2003). Nurses and the virtues of dealing with existential questions in palliative care. Nursing Ethics, 10(4), 377-387.

Hunt, M.R. et Carnevale, F.A. (2011). Moral experience: a framework for bioethics research. Journal of Medical Ethics, 37(11), 658-662.

Jacono, B., Cable-Williams, B., Foster, C., Mitchell, M.G., Lavoie, M., Richardson, H., ... Baxter, S. (2009). Principes et pratiques des soins infirmiers palliatifs et compétences en soins palliatifs à lintention du personnel infirmier au Canada. Consulté sur http://casn.ca/wp-content/uploads/2014/12/ MicrosoftWordCompetenciesDocumentFinalFR.pdf

Lincoln, Y. et Guba, E. (1985). Naturalistic Inquiry. Beverly Hills: Sage.

Mak, Y.Y.W. et Elwyn, G. (2005). Voices of the terminally ill: Uncovering the meaning of desire for euthanasia. Palliative Medicine, 19(4), 343-350.

Matzo, M.L. et Schwarz, J.K. (2001). In their own words: Oncology nurses respond to patient requests for assisted suicide and euthanasia. Applied Nursing Research, 14(2), 64-71.

Mok, E. et Chiu, P.C. (2004). Nurse-patient relationships in palliative care. Journal of Advanced Nuring, 48(5), 475-483.

Müller-Busch, H.C. (2015). Issues of palliative medicine in end-of-life care. Dans C. Rehmann-Sutter, H. Gudat et K. Ohnsorge (Éd.), The Patient's Wish to Die: Research, Ethics, and Palliative Care. Oxford : Oxford University Press.

Nortvedt, P. (2001). Needs, closeness and responsibilities. An inquiry into some rival moral considerations in nursing care. Nursing Philosophy, 2(2), 112-121.

Norwood, F. (2007). Nothing more to do: euthanasia, general practice, and end-of-life discourse in the Netherlands. Medical Anthropology, 26(2), 139-174.

Ohnsorge, K., Gudat Keller, H.R., Widdershoven, G.A.M. et Rehmann-Sutter, C. (2012). 'Ambivalence' at the end of life: How to understand patients' wishes ethically. Nursing Ethics, 19(5), 629-641.
Onwuteaka-Philipsen, B.D., Brinkman-Stoppelenburg, A., Penning, C., de Jong-Krul, G.J.F., van Delden, J.J.M. et van der Heide, A. (2012). Trends in end-of-life practices before and after the enactment of the euthanasia law in the Netherlands from 1990 to 2010: A repeated cross-sectional survey. Lancet, 380(9845), 908-915.

Oregon Public Health Division (2013). Oregon's Death with Dignity Act-2013. Consulté sur : http://public.health. oregon.gov/ProviderPartnerResources/EvaluationResearch/ DeathwithDignityAct/Documents/year16.pdf

Publications Québec, 2016. Projet de loi no 52 : Loi concernant les soins de fin de vie, 40e législature, 1re session. Consulté sur http:// legisquebec.gouv.qc.ca/fr/ShowDoc/cs/S-32.0001

Raus, K., Brown, J., Seale, C., Rietjens, J.A.C., Janssens, R., Bruinsma, S., ... Sterckx, S. (2014). Continuous sedation until death: the everyday moral reasoning of physicians, nurses and family caregivers in the UK, The Netherlands and Belgium. BioMed Central Medical Ethics, 15(1), 14-24.

Rietjens, J.A., van der Maas, P.J., Onwuteaka-Philipsen, B.D., van Delden, J.J. et van der Heide, A. (2009). Two decades of research on euthanasia from the Netherlands. What have we learnt and what questions remain. Journal of Bioethical Inquiry, 6(3), 271-283.

Roberts, D. et Snowball, J. (1999). Psychological care in oncology nursing: a study of social knowledge. Journal of Clinical Nursing, $8(1), 39-47$.

Schwarz, J.K. (2003). Understanding and responding to patients' requests for assistance in dying. Journal of Nursing Scholarship, 35(4), 377-384.

Schwarz, J.K. (2004). Responding to persistent requests for assistance in dying: A phenomenological inquiry. International Journal of Palliative Nursing, 10(5), 225-235.

Strang, S., Henoch, I., Danielson, E., Browall, M. et MelinJohansson, C. (2014). Communication about existential issues with patients close to death - nurses' reflections on content, process and meaning. Psychooncology, 23(5), 562-568.

Van de Scheur, A. et van der Arend, A. (1998). The Role of Nurses in Euthanasia: A Dutch study. Nursing Ethics, 5(6), 497-508.

Van der Mass, P.J., van Delden, J.J.M., Pignenborg, L. et Looman, C.W.N. (1991). Euthanasia and other medical decisions concerning the end of life. The Lancet, 338(8768), 669-673.

Volker, D.L. (2001). Oncology nurses' experiences with requests for assisted dying from terminally ill patients with cancer. Oncology Nurses Forum, 28(1), 39-49.

Volker, D.L. (2003). Assisted dying and end-of-life symptom management. Cancer Nursing, 26(5), 392-399.

Woods, M. et Ascher, J.B. (2015). Nurses and the euthanasia debate: Reflections from New Zealand. International Nursing Review, 62(1), 13-20.

Wright, D. et Brajtman, S. (2011). Relational and embodied knowing: Nursing ethics within the interprofessional team. Nursing Ethics, 18(1), 20-30.

Wright, D, Brajtman, S. et Bitzas, V. (2009). Human relationships at the end of life: an ethical ontology for practice. Journal of Hospice and Palliative Nursing, 11(4), 219-227.

Wright, D.K. et Pugnaire Gros, C. (2012). Theory inspired practice for end-of-life cancer care: An exploration of the McGill Model of Nursing. Canadian Oncology Nursing Journal, 22(3), 175-181. 